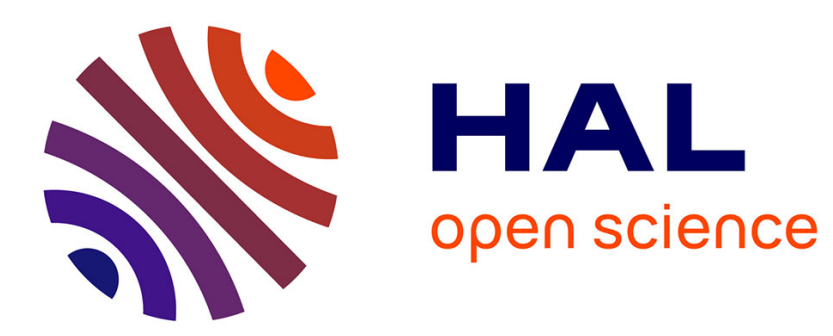

\title{
Manomètre à silastène pour la mesure des hautes pressions dynamiques dans les solides
}

\author{
Armand Rauch
}

\section{To cite this version:}

Armand Rauch. Manomètre à silastène pour la mesure des hautes pressions dynamiques dans les solides. Revue de Physique Appliquée, 1966, 1 (1), pp.68-70. 10.1051/rphysap:019660010106800 . jpa-00242686

\section{HAL Id: jpa-00242686 https://hal.science/jpa-00242686}

Submitted on 1 Jan 1966

HAL is a multi-disciplinary open access archive for the deposit and dissemination of scientific research documents, whether they are published or not. The documents may come from teaching and research institutions in France or abroad, or from public or private research centers.
L'archive ouverte pluridisciplinaire HAL, est destinée au dépôt et à la diffusion de documents scientifiques de niveau recherche, publiés ou non, émanant des établissements d'enseignement et de recherche français ou étrangers, des laboratoires publics ou privés. 


\title{
MANOMĖTRE A SILASTÈNE POUR LA MESURE DES HAUTES PRESSIONS DYNAMIQUES DANS LES SOLIDES
}

\author{
Par Armand RaUCh, \\ Commissariat à l'Énergie Atomique.
}

Résumé. - Le silastène est un corps siliconé qui reste électriquement isolant jusqu'à des pressions très élevées. Soumis à l'action d'une onde de choc, il présente une résistance mesurable (400 ohms) à partir de 225 kilobars. Au-delà de 360 kilobars, la résistance devient de l'ordre de l'ohm. La décroissance de la résistance suit une loi doublement linéaire, avec un point de transition à 255 kilobars.

Cette propriété du silastène permet de l'utiliser comme manomètre de choc pour un domaine de pressions de 1 à 2 mégabars dans les métaux.

\begin{abstract}
Silastene is a silicone rubber which remains an electrical insulator even under high pressure. At a pressure of 225 kilobars induced by a shock wave, its electrical resistance can be measured (about $400 \Omega$ ). Beyond 360 kilobars its resistance falls to under $1 \Omega$. The decay of the resistance obeys two linear laws: The first one up to $255 \mathrm{~Kb}$ and the second from $255 \mathrm{~Kb}$ to $360 \mathrm{~Kb}$. At the pressure of 255 kilobars there is a transition point.

This property of silastene allows it to be used as a shock manometer for dynamic pressures in metals between one and two megabars.
\end{abstract}

1. Introduction. - Tous les isolants électriques soumis à une haute pression (statique ou dynamique) voient en général leur résistivité décroître. Beaucoup de ces éléments présentent un ou plusieurs points de transitions.

Cette observation a été faite pour un certain nombre de corps simples ou composés [1]. Le phénomène de conduction apparaît en général à des pressions relativement faibles. Pour le sélénium sous pression statique un point de transition apparaît à environ 120 kilobars, où la résistance passe brusquement de $10^{3}$ à $1 \Omega$. Sous pression dynamique (compression par choc) cette limite est notablement abaissée.

Pour l'iode on décèle sous pression statique une conduction à partir de 100 kilobars et une discontinuité à 250 kilobars, alors que sous pression dynamique l'iode commence à conduire aux environs de 20 kilobars, et que la discontinuité se situe à 40 kilobars.

2. Conduction du silastène sous choc. - Nous avons étudié la variation relative de résistance d'un échantillon de silastène ${ }^{(1)}$ en fonction de la pression dynamique. L'échantillon de silastène est monté sur un disque de cuivre dans lequel on induit une onde de choc intense à l'aide d'une autre plaque de cuivre projetée par une cartouche d'explosif. On mesure à l'aide de contacts électriques la vitesse que prend la surface libre de la cible en cuivre sous l'effet de l'onde de choc. La pression dynamique est

( $\left.{ }^{1}\right)$ Silastène RTV 501 de la Société industrielle des Silicones.

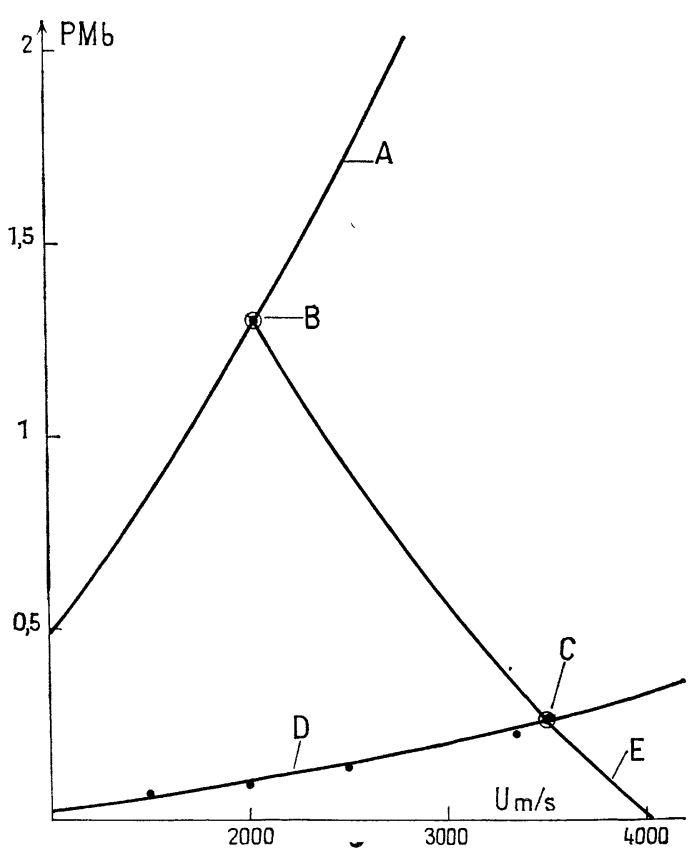

FIG. 1. - $A$ : polaire de choc du cuivre $\rho_{0}=8,89$

$B:$ pression de choc dans le cuivre

$C:$ pression de choc dans le silastène

$D:$ polaire de choc du silastène $\rho_{0}=1,13$

$E:$ vitesse de surface libre de la cible en cuivre.

reliée à la vitesse de surface libre par une loi caractéristique de chaque corps, et qui, pour le cuivre est connue [2]. D'autre part, on mesure la résis- 
tance électrique de l'échantillon de silastène au passage de l'onde de choc. Cette expérience est répétée à des pressions différentes en faisant varier la vitesse du projectile de cuivre. On passe de la connaissance de la pression dans le cuivre à celle dans le silastène à l'aide de leurs polaires de choc respectives (voir $f i g$. 1) et en appliquant les lois de l'onde de choc. Nous avons déterminé celle du silastène à l'aide d'expériences annexes dont le principe est décrit en [2].

La figure 2 indique l'arrangement expérimental ct la courbe de la résistance en fonction de la pression. Les tensions $V_{1}$ et $V_{2}$ mesurent respectivement l'in-

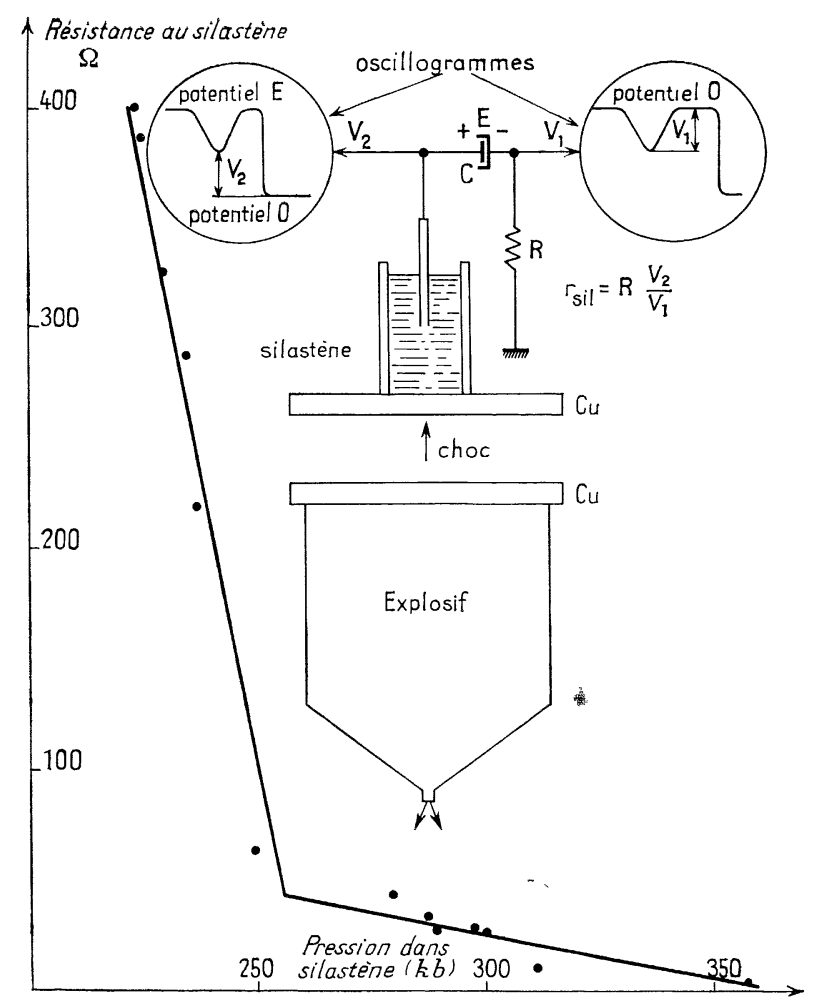

FIG. 2.

tensité du courant et la différence de potentiel aux bornes de l'échantillon. L'énergie électrique est fournie par un condensateur électrochimique C placé à proximité immédiate du dispositif de façon à réduire la self cu circuit. La figure 3 représente deux enregistrements photographiques de la tension $V_{2}$ obtenues à deux pressions différentes.

Le premier signal est dû à la conduction sous choc du silastène. La chute rapide vers la tension zéro, qui suit le premier signal, est due au court-circuit apparaissant entre les électrodes lorsque la plaque de cuivre, mise en mouvement par le choc, atteint l'électrode centrale.

Pour une pression inférieure à 225 kilobars, on ne décèle pas de variation notable de la résistance. Par contre, au-dessus de cette valeur elle décroît avec une pente de $100 \Omega$ pour 10 kilobars. A 225 kilobarson observe un changement de pente et une décroissance de l'ordre de $2 \Omega$ pour 10 kilobars de pression. Au-dessus de 360 kilobars, la résistance est trop faible pour être mesurable.
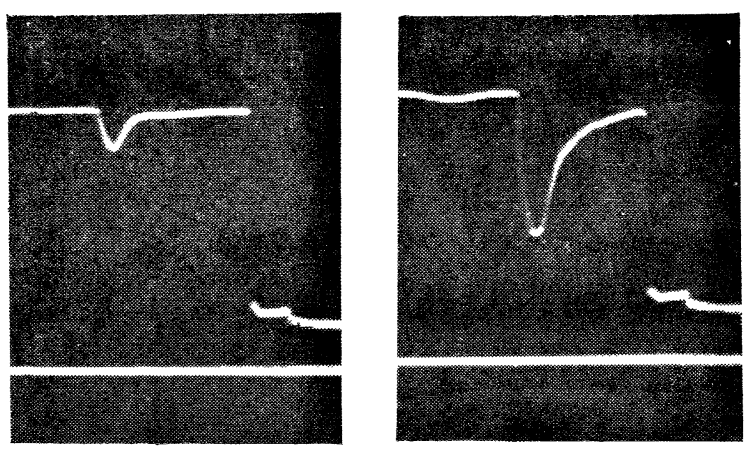

FIg.3. - à gauche: Pression de 1,18 mégabar dans le cuivre à droite : Pression de 1,52 mégabar dans le cuivre.

Le changement de pente est sans doute dû au passage d'un état semiconducteur à un état métallique du silastène.

A notre connaissance, le silastène est le seul élément qui passe de l'état isolant à l'état conducteur à une pression aussi élevée.

Cette constatation nous a permis de réaliser un manomètre utilisable pour la mesure des pressions dynamiques entre 225 et 360 kilobars dans le silastène, soit entre 1,1 et 1,7 mégabars dans le cuivre.

3. Réalisation du manomètre. - Le schéma électrique et les cotes du manomètre sont donnés par la figure 4.

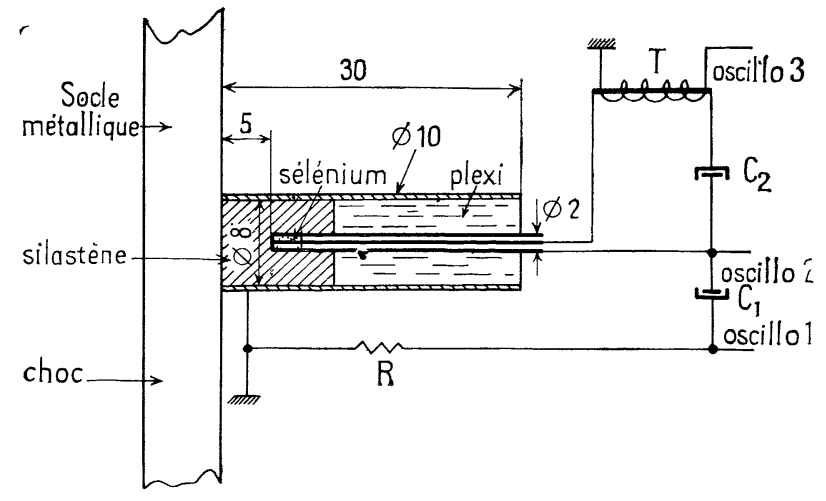

FIG. 4.

La conduction électrique se fait entre l'électrode centrale $(\varnothing=2 \mathrm{~mm})$ et l'électrode extérieure $(\varnothing=8 \mathrm{~mm})$. R est une résistance étalonnée $(50 \Omega)$. 
L'oscillographe 1 mesure le courant électrique dans l'échantillon et l'oscillographe 2 mesure la tension aux bornes des électrodes.

Dans deux cas on n'observe qu'un court-circuit franc sur les oscillographes 1 et 2 : soit pour une pression inférieure à 225 kilobars, soit pour une pression supérieure à 360 kilobars. En effet, dans le premier cas, le silastène ne devient pas conducteur, mais on enregistre quand même le court-circuit dû au déplacement de la plaque métallique. Dans le deuxième cas, la résistance du silastène est très faible et apparaît donc comme un court-circuit.

Pour permettre de discerner ces deux cas, l'électrode centrale est munie intérieurement d'une électrode coaxiale isolée de la première par du sélénium. Le sélénium devient conducteur à une faible pression de l'ordre de 60 kilobars. Lorsque l'onde de choc passe à travers le sélénium et le transformateur T. L'oscillographe 3 enregistre l'instant de ce passage. Si la pression de choc dans le silastène est inférieure au seuil de 225 kilobars, le passage de l'onde de choc ne fera apparaître de signal que sur l'oscillographe 3 . Les signaux sur les oscillographes 1 et 2 , liés seulement au court-circuit par la plaque métallique apparaîtront quelques microsecondes plus tard.

Par contre, si la pression est supérieure à 360 kilobars, les résistances du sélénium et du silastène tombent à zéro dès le passage de l'onde et les trois signaux seront synchrones.

4. Conclusion. - Ce manomètre n'est pas aussi précis que les moyens habituels pour la mesure de la pression, c'est-à-dire les mesures de vitesse de choc ou de vitesse de surface libre d'échantillons soumis au choc. Mais la simplicité de sa mise en œuvre peut le faire préférer à d'autres moyens, surtout si une connaissance très précise de la pression n'est pas exigée.

La courbe d'étalonnage dépend de la géométrie du manomètre. Par contre le point de transition en est indépendant.

Manuscrit reçu le 8 octobre 1965.

\section{RÉFÉRENCE 1}

SUR LE SÉLÉNIUM :

Monten, Arkiø Math. Astron. Och. Fysik, 1909, 4, No 31,16

Bridgmann, Proc. Amer. Acad. Sc., 1952, 81.

Drickammer, Congrès de la Fédération européenne du Génie Chimique, Londres, juin 1962.

Thounenin (J.) et Rauch (A.), C.R. Acad. Sc., 1962, 255,868 .

SUR L'iode ET PHOSPHORE ROUGE :

Alder (B. J.) et Christian (R. H.), Phys. Rev., 1956, 104, 550 .
SUR LE SOUfRE :

Havid (H. G.) et Hamann (S.), J. Chem. Physics, 1958, 28, 1006

Joigneau (S.) et Thouvenin (J.), C.R. Acad. Sc., 1958, 246, 3422 .

Sur L'eau :

Brish (A. A.), Tarason (M. S.) et Tsuk ermann (V. A.), J. Exptl. Theoret. Phys. (U. S. S. R.), 1960, 38, $22-25$.

\section{RÉFÉRENCE 2}

Walsh, Rice et McQuenn, Solide State Physics, vol. 6, p. 1 et suivantes, Academic Press, New-York. 